\title{
Assistência de enfermagem à saúde da mulher na Atenção Básica: uma revisão da
}

\section{literatura}

\author{
Nursing care for women's health in Primary Care: a literature review \\ Cuidados de enfermería para la salud de la mujer en la Atención Primaria: una revisión de la
} literatura

\author{
Maria Gabriela de Oliveira Frazão \\ ORCID: https://orcid.org/0000-0002-5223-3493 \\ Universidade Maurício de Nassau, Brasil \\ E-mail: gabriela.oliveirafz@gmail.com \\ Ester Teixeira Lopes \\ ORCID: https://orcid.org/0000-0002-9894-5760 \\ Universidade Maurício de Nassau, Brasil \\ E-mail: ester17teixeira@gmail.com \\ Sabrina Iracema da Silva Couto \\ ORCID: https://orcid.org/0000-0002-3864-4843 \\ Universidade Maurício de Nassau, Brasil \\ E-mail: sabrinacouto89@gmail.com \\ Larissa Gabriele Farias e Silva \\ ORCID: https://orcid.org/0000-0001-8235-8310 \\ Universidade Maurício de Nassau, Brasil \\ E-mail: larissagabrielefariasesilva@gmail.com \\ Maria Gabriela Cristina Pereira Mousinho \\ ORCID: https://orcid.org/0000-0002-1479-2008 \\ Universidade Maurício de Nassau, Brasil \\ E-mail: mgabird@gmail.com \\ Milena Catarine Silva de Araújo \\ ORCID: https://orcid.org/0000-0002-3577-9873 \\ Universidade Maurício de Nassau, Brasil \\ E-mail: milenaaraujoo45@gmail.com \\ Danielly Danubia da Silva \\ ORCID: https://orcid.org/0000-0002-5396-8002 \\ Universidade Maurício de Nassau, Brasil \\ E-mail: daniellydanubia2016@outlook.com \\ Alicia Nathalia Lemos de Melo \\ ORCID: https://orcid.org/0000-0002-0340-7004 \\ Universidade Maurício de Nassau, Brasil \\ E-mail: alicianathalia2016@hotmail.com \\ Dayana Ruthyally da Rocha Silva \\ ORCID: https://orcid.org/0000-0003-1982-0482 \\ Universidade Maurício de Nassau, Brasil \\ E-mail: ruthyallyd@gmail.com \\ Diego Wictor da Silva \\ ORCID: https://orcid.org/0000-0001-7085-3218 \\ Universidade Maurício de Nassau, Brasil \\ E-mail: diegowictorr@gmail.com \\ Laryssa Grazielle Feitosa Lopes \\ ORCID: https://orcid.org/0000-0002-0709-5378 \\ Universidade Maurício de Nassau, Brasil \\ Faculdade de Medicina do Sertão, Brasil \\ E-mail: lara_grazi@hotmail.com
}

\section{Resumo}

Objetivo: Relatar com base na literatura científica como é realizada a assistência de enfermagem à saúde da mulher na atenção básica, buscando descrever seus benefícios e discutindo seu funcionamento. Metodologia: Trata-se de uma Revisão Integrativa da Literatura de cunho exploratório e quantitativo, realizada nas bases de dados: Medical Literature Analysis and Retrieval System Online (MEDLINE), Literatura Latino-Americana e do Caribe em Ciências da Saúde (LILACS), Scientific Electronic Library Online (SCIELO) e Banco de Dados em Enfermagem (BDENF), foram utilizados artigos escritos no idioma português, publicados no período entre 2017 e 2021. Resultados: Foram 
encontrados 537 artigos no total, após rigor metodológico por meio dos critérios de inclusão e exclusão, foram selecionados 11 artigos, sendo 0 na MEDLINE, 5 na LILACS, 0 na SCIELO e 6 na BDENF. A apuração das informações foi feita de forma descritiva e predispôs a etapa de extração dos dados: autor, título, tipo de estudo, objetivo e ano de publicação. Conclusão: O estudo evidenciou que na atenção básica a enfermagem mostra uma influência indispensável, que de acordo com as normativas e políticas atribuídas aos enfermeiros sobre seu caráter assistencial, ético e de gerenciamento existe questões de senso comum que os profissionais devem compor para garantir o cuidado contínuo.

Palavras-chave: Saúde da mulher; Assistência de enfermagem; Atenção básica.

\begin{abstract}
Objective: To report, based on the scientific literature, how nursing care for women's health is carried out in primary care, seeking to describe its benefits and discussing its functioning. Methodology: This is an integrative literature review of an exploratory and quantitative nature, carried out in the following databases: Medical Literature Analysis and Retrieval System Online (MEDLINE), Latin American and Caribbean Literature on Health Sciences (LILACS), Scientific Electronic Library Online (SCIELO) and Nursing Database (BDENF), articles written in Portuguese, published between 2017 and 2021 were used. Results: A total of 537 articles were found, after methodological rigor through the inclusion criteria and exclusion, 11 articles were selected, 0 in MEDLINE, 5 in LILACS, 0 in SCIELO and 6 in BDENF. The verification of the information was done in a descriptive way and predisposed the stage of data extraction: author, title, type of study, objective and year of publication. Conclusion: The study showed that in primary care, nursing shows an indispensable influence, that according to the regulations and policies assigned to nurses about their care, ethical and management character, there are common sense issues that professionals must compose to guarantee care continuous.
\end{abstract}

Keywords: Women's health; Nursing assistance; Basic attention.

\begin{abstract}
Resumen
Objetivo: Relatar, con base en la literatura científica, cómo se realiza el cuidado de enfermería a la salud de la mujer en la atención primaria, buscando describir sus beneficios y discutir su funcionamiento. Metodología: Se trata de una revisión integradora de la literatura de carácter exploratorio y cuantitativo, realizada en las siguientes bases de datos: Medical Literature Analysis and Retrieval System Online (MEDLINE), Latin American and Caribbean Literature on Health Sciences (LILACS), Scientific Electronic Library Online (SCIELO) y Base de Datos de Enfermería (BDENF), se utilizaron artículos escritos en portugués, publicados entre 2017 y 2021 . Resultados: Se encontraron un total de 537 artículos, luego del rigor metodológico a través de los criterios de inclusión y exclusión, se seleccionaron 11 artículos, 0 en MEDLINE, 5 en LILACS, 0 en SCIELO y 6 en BDENF. La verificación de la información se hizo de forma descriptiva y predispuso la etapa de extracción de datos: autor, título, tipo de estudio, objetivo y año de publicación. Conclusión: El estudio mostró que en la atención primaria, la enfermería muestra una influencia indispensable, que de acuerdo con las normas y políticas asignadas a las enfermeras sobre su carácter asistencial, ético y de gestión, existen cuestiones de sentido común que los profesionales deben componer para garantizar la continuidad del cuidado.
\end{abstract}

Palabras clave: Salud de la mujer; Asistencia de enfermería; Atención básica.

\title{
1. Introdução
}

O feminismo a partir de Wollstonecraft (2016), evidencia que as mulheres buscam seu espaço na sociedade há muito tempo, pois são associadas a pessoas de sexo frágil, que devem se submeter a certas rotinas cogitadas pela sociedade, onde viveram por muito sobre a sombra do homem, que sempre foram enaltecidos durante toda história social. Buscando seu espaço, o ser mulher vem quebrando paradigmas lutando por direitos, igualdade, valorização no mercado de trabalho e reconhecimentos, tais foram emergidas durante uma longa trajetória fazendo uma desconstrução das diferenças.

Existem políticas voltadas a sanar com estabelecidas adversidades relacionada à saúde, ou dar respostas a problemas que atingem determinados grupos, assim ocorreu em 1984 com o Programa de Assistência Integral à Saúde da Mulher (PAISM), resultado da luta feminista pelo direito das mulheres a terem entrada ao sistema de saúde de forma integral, e não somente sob o cunho reprodutivo (Coelho et al., 2009).

O PAISM oferta assistência para saúde da mulher voltada para a parte do pré-natal, planejamento familiar, parto e puerpério, ginecológico, câncer de colo de útero e de mama, Infecções Sexualmente Transmissíveis (IST), no climatério, aderindo a quaisquer outras necessidades que as mulheres venham a possuir (Brasil, 2004). Em 2003 a Secretaria de Politicas para as Mulheres da Presidência da República (SPM/PR) foi concebida e integrada ao Ministério das Mulheres, dos Direitos 
Humanos e da Igualdade Racial, gerando assim a Secretaria de Políticas para as Mulheres que tem como compromisso construir um país menos desigual, mais justo e democrático incluindo o reconhecimento feminino, implementando políticas públicas para assistência e validação dos direitos femininos (Castro et al., 2015).

A estrutura do capítulo do Plano Nacional de Políticas para as Mulheres (PNPM) é a seguinte: igualdade e autossuficiência monetária no âmbito trabalhista; igualdade e educação cívica; saúde geral das mulheres, direitos reprodutivos e sexuais; lidar com as variadas formas de violência contra as mulheres; promoção de autonomia relacionada a tomada de decisões, direitos e participação; crescimento suportável com correspondência monetária e social; mulheres rurais têm direitos iguais à terra; cultura, esportes, comunicação e mídia; combate ao racismo, sexíssimo, lesbofobia, juventude, mulheres idosas e com deficiência. Igualdade das mulheres (Brasil, 2013).

Pouco mais da metade da população do Brasil é composta de mulheres, sendo essas as grandes usuárias dos serviços de saúde, usufruindo dos serviços para autocuidado, porém ativamente procuram os serviços de saúde para acompanhar filhos, parentes, amigos e até mesmo vizinhos. As mulheres não cuidam apenas da saúde dos familiares, também cuidam da comunidade e seus residentes. A discriminação referente as mulheres sobre as relações trabalho- gestão e o peso das tarefas domésticas agravam o problema. Outras variáveis como raça, etnia e pobreza destacam ainda mais a desigualdade. Os homens tendem a viver menos que as mulheres, mas as mulheres adoecem com mais frequência (Brasil, 2004).

Quando se fala em políticas públicas de saúde baseadas no Sistema Único de Saúde (SUS), o termo "atendimento total/integral" é muito popular. Encontra-se primeiro os termos da teoria em que o sistema se baseia: universalidade, equidade e integridade. Em concordância com os materiais de gestão municipal da saúde, o primeiro afirma que é necessário garantir que todas as pessoas tenham acesso às ações e serviços de saúde; a equidade visa reduzir a desigualdade e investir mais nos locais onde as necessidades são maiores; a integralidade visa considerar as pessoas como um todo e atender todas as suas necessidades, incluindo a promoção da saúde, prevenção e controle de doenças, tratamento e reabilitação. Ao mesmo tempo, o princípio da integridade pressupõe a integração da saúde com as demais políticas públicas para garantir que sejam realizadas ações intersetoriais entre as diferentes áreas relacionadas à saúde pessoal e à qualidade de vida (Medeiros \& Guareschi, 2009).

A Secretaria de Política para as Mulheres (SPM) tem o compromisso de ofertar serviços mais justos, humanos e eficientes na PNAISM. Ela acredita que a prioridade deve ser: incluir questões de integridade de gênero e identidade étnica no treinamento de profissionais de saúde; dar prioridade às características de mulheres lésbicas, transexuais e mulheres de rua; e acesso a suprimentos de proteção contra doenças sexualmente transmissíveis HIV/AIDS, métodos anticoncepcionais e Citopatologia para prevenir o câncer de colo uterino (Brasil, 2018).

O objetivo do aconselhamento de enfermagem ginecológica não é apenas prestar assistência biológica à mulher, mas também articulá-la com os aspectos sociais e psicológicos, de modo a garantir que a assistência prestada seja interdisciplinar, inovadora, transformadora e completa (Catafesta et al., 2015). Infelizmente, é evidenciado que muitas mulheres não procuram atendimento precocemente, procurando atendimento médico quando já estão com alguma patologia instalada. Apesar dos esforços realizados, o Brasil ainda não possui uma estratégia eficaz relacionada à prevenção de doenças, influenciado pelo modelo biomédico curativista, voltado para o tratamento e não prevenção (Diniz et al., 2013).

Independente de qual seja o problema de saúde que a mulher possua, a identificação precoce é indispensável, a atenção básica é a porta de entrada para os serviços recomendados à saúde da mulher, seja na área da saúde sexual ou reprodutiva. Os serviços são realizados por enfermeiros ou médicos, favorecendo estes acerca da atenção à saúde da mulher independente de sua sexualidade (de Araujo et al., 2019). É recomendado que o enfermeiro tenha a visão do ser humano como seres individualizados, com histórias pregressas únicas, com valores e crenças definidas a partir de suas culturas. Tendo essa visão o enfermeiro torna-se participante ativo na educação em saúde, conseguindo assim se aproximar das pacientes de jeito a 
descobrirem padrões únicos para cada uma, ao invés de usar um padrão único estereotipado pela literatura, mídia e/ou sociedade (Garcia \& Lisboa, 2012).

Desse modo, diante da urgência em ter um profissional capacitado na atenção básica, faz-se necessária a produção acadêmica de trabalhos científicos que tratem da assistência de enfermagem na atenção básica. Logo, o estudo tem como objetivo central relatar com base na literatura científica como é realizada a assistência de enfermagem à saúde da mulher na atenção básica, buscando descrever os seus benefícios e discutindo seu funcionamento.

\section{Metodologia}

Este estudo trata-se de uma Revisão Integrativa da Literatura, de cunho exploratório e quantitativo. Esta modalidade de revisão é resultado da análise criteriosa de pesquisas, observando-se resultados que compõem o melhor desenvolvimento da pratica, através do conhecimento adquirido, a qual tem a seguinte ordem: 1- formulação da pergunta norteadora, 2 especificação dos métodos e busca da amostragem na literatura, 3- extração dos dados, 4- análise e avaliação dos estudos incluídos, 5- discussão dos resultados, 6- finalização da revisão integrativa (Souza et al., 2010; Botelho et al., 2011).

Para conduzir esta pesquisa utilizou-se a seguinte questão norteadora: Como ocorre a assistência de enfermagem à saúde da mulher na atenção básica?

A coleta de dados ocorreu durante o mês de outubro de 2021, nas bases de dados eletrônicos: Medical Literature Analysis and Retrieval System Online (MEDLINE), Literatura Latino-Americana e do Caribe em Ciências da Saúde (LILACS), Scientific Electronic Library Online (SCIELO) e Banco de Dados em Enfermagem (BDENF). Para as bases de dados foram utilizados os seguintes Descritores em Ciências da Saúde (DeCS): "Saúde da Mulher", "Assistência de Enfermagem”, "Atenção Básica”, realizando combinações entre eles para maior busca de artigos.

Foram utilizados como critérios de inclusão: estudos que tratassem da assistência de enfermagem à saúde da mulher na atenção básica, no espaço temporal de 2017-2021, apresentados em textos completos e gratuitos, no idioma português. Foram utilizados como critério de exclusão: capítulos de livros, artigos incompletos, duplicados e sem referência a temática abordada.

\section{Resultados}

Foram encontrados 537 artigos no total, após rigor metodológico com os critérios de inclusão e exclusão, foram selecionados 11 artigos para estudo, sendo 0 na MEDLINE, 5 na LILACS, 0 na SCIELO e 6 na BDENF. A forma de seleção descrita pode ser observada na Figura 1. 
Figura 1: Fluxograma da forma de seleção dos artigos.

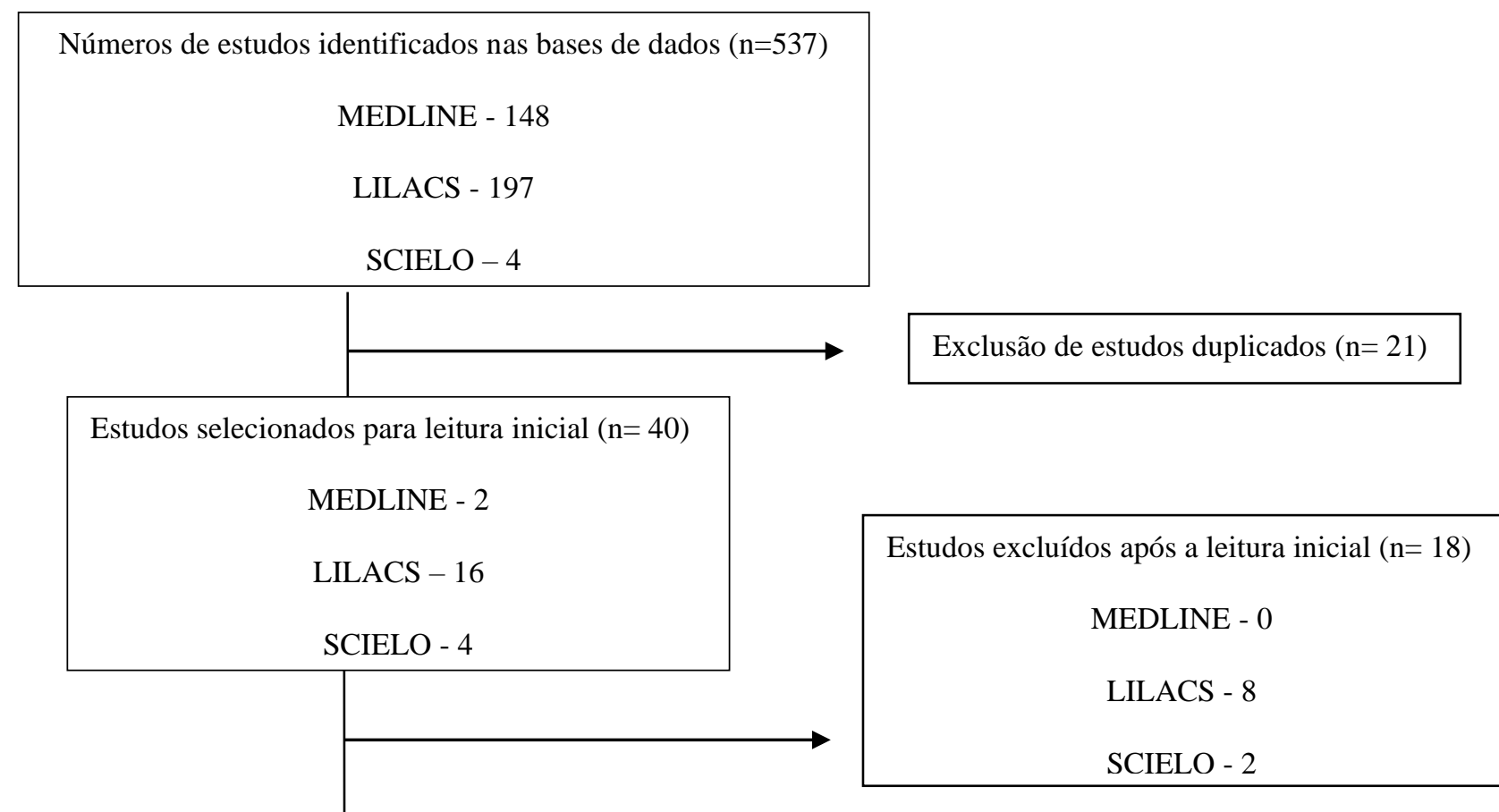

Estudos selecionados para leitura na íntegra e avaliação de elegibilidade $(n=22)$ :

MEDLINE -2

LILACS -8

SCIELO - 2

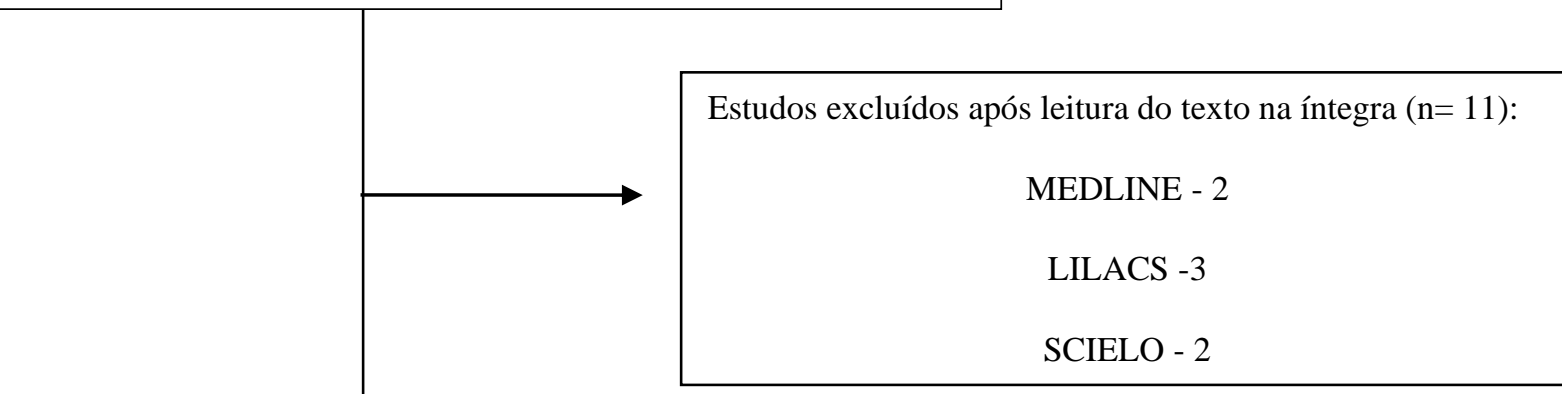

Estudos Incluídos na revisão $(\mathrm{n}=11)$ :

$$
\begin{aligned}
& \text { MEDLINE - } 0 \\
& \text { LILACS - } 5 \\
& \text { SCIELO - } 0
\end{aligned}
$$

Fonte: Autores (2021).

Após criteriosa análise dos artigos selecionados, a apuração das informações foi feita de forma descritiva e predispôs a etapa de extração dos dados: autor, título, tipo de estudo, objetivo e ano de publicação (Quadro 1). 
Quadro 1: Resultados dos artigos selecionados.

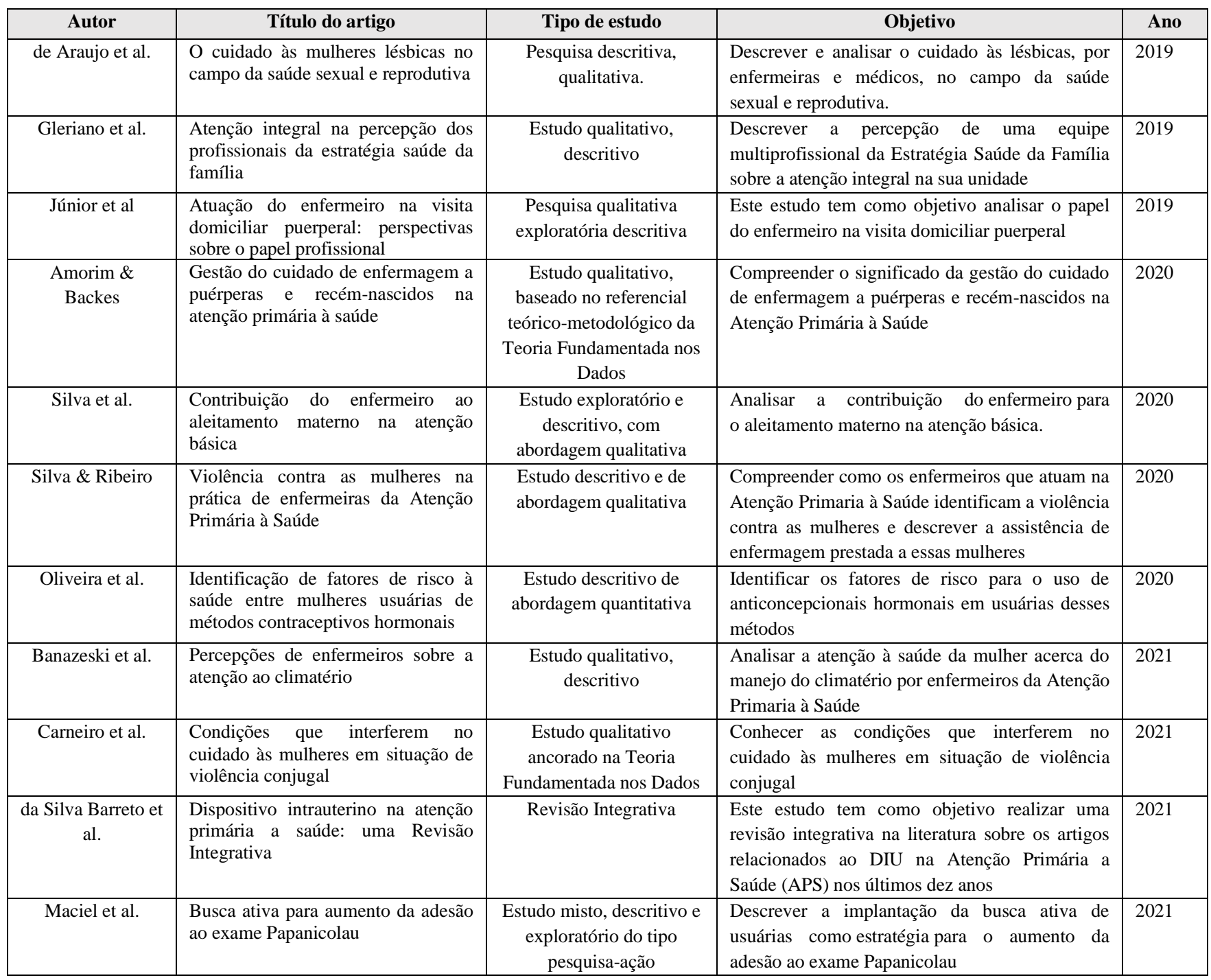

Fonte: Autores (2021).

Analisando os dados do quadro, percebe-se que os artigos trabalhados têm prevalência entre os anos de 2019-2021, dando resultados atualizados.

\section{Discussão}

De acordo com Júnior et al. (2019) a essência da profissão de enfermagem é o cuidado com ser humano. Tendo seu papel reconhecido por sua habilidade e capacidade em compreender e executar o cuidado, observando o indivíduo como um todo. Como integrante da Atenção Primaria de Saúde (APS), o enfermeiro propicia o contato e interação entre os usuários, comunidade e profissionais, buscando aprimorar os contatos e as ações de cuidado em saúde. A enfermagem é responsável pela liderança de sua equipe e gerenciamento de conclusões, centrada na atuação participativa interpessoal, desempenhando uma função de fator organizacional, definindo uma identidade de autonomia (Umpiérrez, 2013).

Os profissionais através da educação em saúde podem fornecer informações e métodos que podem auxiliar no autocuidado, o encorajamento dos usuários para procurar as unidades de saúde das suas localidades para fazer um acompanhamento contínuo também é papel do enfermeiro (Ohl et al., 2016). Para se ofertar o cuidado adequado a mulher, o profissional de enfermagem deve compreender as variadas vivências envolvidas (Silva \& Ribeiro, 2020). 
Para abranger a qualidade dos serviços de forma eficaz na atenção básica, a HumanizaSUS foi instituída pelo ministério da saúde em 2003, onde busca a junção de práticas que fortaleçam a coletividade do atendimento e de gestão em todos os setores, passando assim a dar assistência e reconhecimentos dos direitos de saúde aos cidadãos (Brasil, 2008).

Ao decorrer do tempo as mulheres conquistaram espaço na sociedade e passaram a ter mais direitos e acesso, no que condiz a serviços de saúde, os fatores que prejudicam a busca por esses serviços por mulheres é proveniente de violações de direitos, relutância ao atendimento, favorecendo assim para um mau acompanhamento e não garantindo uma prestação e promoção de saúde adequados (Brasil, 2004). Com a instalação do PAISM, os planos assistenciais sobre os ciclos da gravidez, pré-natal, parto e puerpério, educação em saúde acerca do autoexame de mama e exame de rotina de câncer de colo de útero, autocuidado e sua importância, aconselhamento sobre gravidez na adolescência e sobre seus riscos, prevenção e tratamento de infecções sexualmente transmissíveis (ISTs) adquiridas pelo sexo sem proteção (D'Oliveira, 1999).

Os cuidados de enfermagem à mulher como gestante ou puérpera, seus filhos ou familiares acontece de forma prevalente na atenção primária à saúde e por enfermeiros. No que se refere à saúde da mulher no ciclo gravídico, em estudo realizado por Amorim e Backes (2020), na atenção básica, contou com a cooperação de gestantes que se encontravam no $3^{\circ}$ trimestre gestacional, este estudo evidenciou que ainda existem brechas para uma assistência de qualidade, apesar de mostrarem-se satisfeitas, em tese, as gestantes relacionaram a qualidade da assistência recebida, ao fato de como foram acolhidas e tratadas, e não a assistência clínica.

Os autores Silva et al. (2020), observaram que o enfermeiro tem papel importante e fundamental na orientação acerca do aleitamento materno no contexto da atenção básica, desenvolvendo promoções e ações já no pré natal e prolongando até a visita puerperal, oportunizando as consultas durante o período gestacional para estimular a prática da amamentação, elucidando dúvidas sobre os benefícios do aleitamento, desde o desenvolvimento da criança até o laço materno afetivo. $\mathrm{O}$ enfermeiro deve basear as suas orientações na realidade da mulher puérpera, levando em conta suas vivências culturais, suas dificuldades e seu potencial, assim como o enfermeiro precisa guiar essas mulheres com base nas perspectivas delas e de seus familiares (Junior et al., 2019).

Conforme relatado por Villela e Arilha (2003), no contexto histórico, por muito tempo, as mulheres eram vistas apenas pelo aspecto reprodutivo, tendo uma percepção de mulher do lar. A APS tem papel importante no planejamento reprodutivo, sendo essa um campo importante para disponibilizar o Dispositivo Intrauterino (DIU) às mulheres, favorecendo e expandindo o acesso ao serviço. Assim sendo amplificando as opções de métodos contraceptivo, dando as mulheres opções de escolha que mais se aproximem de sua realidade e consoante a sua necessidade (da Silva Barreto et al., 2021).

Em conformidade, Oliveira et al. (2020), também descreve que é relevante que o enfermeiro encoraje às pacientes sobre os métodos de barreira e a dupla proteção, que consiste na combinação de dois métodos protetivos, principalmente para as mulheres com mais de um parceiro sexual, orientando sobre gestações indesejadas bem como a prevenção de contraírem alguma IST, o enfermeiro é personagem principal no empoderamento dos usuários e na educação em saúde.

Por se tratar da principal porta de entrada dos serviços à saúde, a APS tem um papel importante na resolução do adoecer, é onde basicamente se tem primeiro contato com os usuários, tendo em vista o acompanhamento e detecção precoce de patologias, e quando se trata de patologias voltada para saúde da mulher, como a detecção precoce do câncer de colo de útero (CCU), a atenção básica envolve soluções preventivas, auxiliando sobre o autocuidado, assim diminuindo o agravamento de doenças (Guimarães et al., 2012). Assim sendo, a deteç̧ão precoce de alterações do CCU e rastreio de outras doenças ocorre por meio do exame preventivo do câncer de colo do útero (PCCU), a detecção do câncer de mama acontece através do exame clínico das mamas, é de extrema importância que a mulher faça o exame todo ano, ou, consoante a situação de sua saúde, em intervalos mais curtos ou mais extensos (Oliveira et al., 2020). 
É fundamental a busca enérgica das mulheres para o diagnóstico precoce do CCU, o profissional tem a obrigação de aprimorar a adesão ao exame citológico. Esse intermédio indica a consolidação das relações profissionais entre a equipe de saúde tal como os acadêmicos em enfermagem e a comunidade, alcançando o aperfeiçoamento da adesão e qualidade da cobertura do Papanicolau (Maciel et al., 2021). Ainda assim de acordo com de Araujo et al. (2019) o exame preventivo de colo de útero no que se refere às lésbicas, encontra dificuldades na adesão. Enfermeiros e médicos encontram essas dificuldades nas lésbicas com estereótipo masculino, já entre as mulheres lésbicas com estereótipo feminino não se encontram dificuldades.

Conforme Silva e Ribeiro (2020) relataram, em casos de violência contra as mulheres a compreensão do enfermeiro acerca do que se passa é muito importante, para assim ofertar o cuidado que atenda às suas reais deficiências. O profissional de saúde pode exercer papel positivo ou negativo no cuidado à mulher em situação de violência, de modo que ele pode, ou não, construir vínculos com essas usuárias (Carneiro et al., 2021). Sendo assim, muitas mulheres lésbicas apresentam fragilidades originadas de suas próprias crenças e outras que sucedem das discriminações e preconceitos experimentados nos serviços de saúde. É verdade que ocorre uma desatenção por parte dos profissionais, frente às necessidades de saúde expressada por essas mulheres, que vivem com a violência simbólica, disposto ao baixo acesso aos bens e serviços de saúde por lésbicas (de Araujo et al., 2019).

Conforme Banazeski et al. (2021), identificou, por meio de uma pesquisa sobre o climatério, o atendimento a atenção a saúde da mulher em climatério, resume-se em um atendimento superficial, baseado em sinais e sintomas e algumas vezes na experiência pessoal do profissional. Foi identificado que a conduta profissional nessa fase não tem embasamento científico rigoroso, e não existe a educação permanente em saúde ofertada pela gestão dos serviços.

De acordo com os princípios pressupostos, é preconizado uma rede assistencial capaz de captar precocemente problemáticas que possam agravar a saúde, assim devendo levar em consideração uma estrutura apta e uma equipe capacitada (Brasil, 2004). No entanto Gleriano et al. (2019) relatou que atenção à saúde da mulher acerca das políticas públicas, da organização dos serviços e das práticas dos profissionais de saúde na APS teve foco maior voltado a mulher no ciclo gravídico, tendo como prioridade o pré-natal, com reduzida valorização do cuidado integral, como planejamento familiar, PCCU, violência contra mulher e climatério.

Neste caso, é urgente o respeito à mulher e as particularidades e singularidade do seu ciclo de vida independente da sua especificidade, tendo seu direito às demandas biológicas, psicológicas e sociais assegurado e resolvido, com respeito e dignidade, preservando a sua autonomia como usuárias dos serviços de saúde acerca do processo saúde doença (Catafesta et al., 2015).

\section{Conclusão}

O estudo evidenciou que na atenção básica a enfermagem mostra uma influência indispensável, que de acordo com as normativas e políticas atribuídas aos enfermeiros sobre seu caráter assistencial, ético e de gerenciamento existe questões de senso comum que os profissionais devem compor para garantir o cuidado contínuo.

A principal porta de entrada de saúde, a atenção básica, tem uma variedade de assistências disponíveis assim como de problemáticas, portanto, é importante que haja uma equipe de enfermagem qualificada para que de acordo com essas problemáticas saibam desenvolver métodos afim implementar medidas que agregue na qualidade de saúde.

Mediante as percepções relacionadas a assistência na enfermagem no que diz respeito a saúde da mulher na atenção básica, é imprescindível que as unidades disponham de estratégias para a adesão dos serviços, métodos como a educação em saúde acerca do autocuidado, auxiliando e definindo sobre a sua importância. Nota-se que nas unidades básicas os profissionais atribuem suas ações voltadas mais para o ciclo gravídico, que é o pré-natal, parto e puerpério, assim reduzindo a atenção a outros serviços importante da saúde da mulher, como o planejamento familiar e o cuidado relacionado a outras patologias que 
podem afetar a qualidade de vida da mulher, como o câncer de colo de útero, câncer de mama, problemas psíquicos e doenças sexuais e crônicas. No entanto, o acolhimento é uma importante ferramenta na consulta em enfermagem, que além de aproximar o profissional-paciente evidencia a importância do seu papel no cuidado, pois através disso se orienta, elabora e implementa medidas que tendem a ajudar as usuárias em qualquer momento de sua vida, independente de idade, raça, etnia, credo e renda, bem como todo seu conjunto familiar.

Acredita-se que esse estudo tenha o teor de fornecer um pensamento resolutivo sobre a assistência em enfermagem na atenção básica e sua importância nos agravos de saúde que norteiam a saúde da mulher.

Desta forma, se faz necessário que estudos futuros sejam desenvolvidos nesta temática, visto que é um assunto importante para a saúde pública, que impacta diretamente no bem-estar das mulheres, e especialmente porque o conhecimento e divulgação desse tema precisa ser difundido amplamente na literatura brasileira.

\section{Referências}

Amorim, T. S., \& Backes, M. T. S. (2020). Gestão do cuidado de enfermagem a puérperas e recém-nascidos na Atenção Primária à Saúde. Universidade Federal do Ceará, 1.

Banazeski, A. C., Luzardo, A. R., Rozo, A. J., \& Sinski, K. C. (2021). Percepções de enfermeiros sobre a atenção ao climatério. J Enfermagem UFPE on line, 15 , e245748.

Botelho, L. L. R., de Almeida Cunha, C. C., \& Macedo, M. (2011). O método da revisão integrativa nos estudos organizacionais. Gestão e sociedade, 5(11), 121-136.

Brasil. (2018). Saúde Integral da Mulher. Ministério da Mulher, da Família e dos Direitos Humanos. https://www.gov.br/mdh/pt-br/navegue-portemas/politicas-para-mulheres/arquivo/assuntos/saude-integral-da-mulher

Brasil. (2004). Secretaria de Políticas para Mulheres - SPM. Ministério da Mulher, da Família e dos Direitos Humanos. https://www.gov.br/mdh/ptbr/navegue-por-temas/politicas-para-mulheres/arquivo/arquivos-diversos/sobre/spm

Brasil. (2008). HumanizaSUS: documento base para gestores e trabalhadores do SUS. Ministério da Saúde. 4, (72).

Brasil. (2017). Infecções Sexualmente Transmissíveis (IST): o que são, quais são e como prevenir. Ministério da Saúde. https://antigo.saude.gov.br/saude-dea-z/infeccoes-sexualmente-transmissiveis-ist

Brasil. (2004). Política Nacional de Atenção Integral à Saúde da Mulher: Princípios e Diretrizes. Ministério da Saúde. Secretaria de Atenção à Saúde, Departamento de Ações Programáticas Estratégicas. https://conselho.saude.gov.br/ultimas_noticias/2007/politica_mulher.pdf

Brasil. (2013). Plano Nacional de Políticas para as Mulheres. Presidência da República - Secretaria de Políticas para as Mulheres,114. http://www.mulheres.ba.gov.br/arquivos/File/Publicacoes/PlanoNacionaldePoliticasparaasMulheres20132015.pdf

Carneiro, J. B., Gomes, N. P., de Almeida, L. C. G., Romano, C. M. C., Silva, A. F. D., Webler, N., \& Mauricio, M. D. A. L. L. D. (2021). Condições que interferem no cuidado às mulheres em situação de violência conjugal. Escola Anna Nery, 25.

Castro, L. M. X. D., Simonetti, M. C. M., \& Araújo, M. J. D. O. (2015). Monitoramento e acompanhamento da Política Nacional de Atenção Integral à Saúde da Mulher PNAISM e do Plano Nacional de Políticas para as Mulheres PNPM. In Monitoramento e acompanhamento da Política Nacional de Atenção Integral à Saúde da Mulher PNAISM e do Plano Nacional de Políticas para as Mulheres PNPM (pp. 46-46).

Catafesta, G., Klein, D. P., Canever, B. P., Lazzari, D. D., \& da Silva, E. F. (2015). Consulta de enfermagem ginecológica na estratégia saúde da família. Arquivos de Ciências da Saúde, 22(1), 85-90.

Coelho, E. D. A. C., Silva, C. T. O., Oliveira, J. F. D., \& Almeida, M. S. (2009). Integralidade do cuidado à saúde da mulher: limites da prática profissional. Escola Anna Nery, 13, 154-160.

Cruz, L. M. B. D., \& Loureiro, R. P. (2008). A comunicação na abordagem preventiva do câncer do colo do útero: importância das influências históricoculturais e da sexualidade feminina na adesão às campanhas. Saúde e Sociedade, 17, 120-131.

da Silva Barreto, D., Maia, D. S., Gonçalves, R. D., \& de Sousa Soares, R. (2021). Dispositivo Intrauterino na Atenção Primária a Saúde: uma revisão integrativa. Revista Brasileira de Medicina de Família e Comunidade, 16(43), 2821-2821.

de Araujo, L. M., Penna, L. H. G., Carinhanha, J. I., \& Costa, C. M. A. (2019). O cuidado às mulheres lésbicas no campo da saúde sexual e reprodutiva. Revista Enfermagem UERJ, 27, 34262.

de Mendonça, M. P. F., Pereira, R. J., de Carvalho, S. S. D. S., Barbosa, J. D. S. P., \& Lima, R. N. (2019). Atuação do enfermeiro no diagnóstico precoce da endometriose. Revista Brasileira Interdisciplinar de Saúde.

Diniz, A. S., Xavier, M. B., Braga, P. P., \& Guimarães, E. A. A. (2013). Assistência à saúde da mulher na atenção primária: prevenção do câncer do colo do útero. Revista de APS, 16(3). 
Research, Society and Development, v. 11, n. 2, e25211225655, 2022

(CC BY 4.0) | ISSN 2525-3409 | DOI: http://dx.doi.org/10.33448/rsd-v11i2.25655

D'Oliveira, A. F. P. L. (1999). Saúde e educação: a discussão das relações de poder na atenção à saúde da mulher. Interface-Comunicação, Saúde, Educação, 3, 105-122.

Garcia, O. R. Z., \& Lisboa, L. C. D. S. (2012). Consulta de enfermagem em sexualidade: um instrumento para assistência de enfermagem à saúde da mulher, em nível de atenção primária. Texto \& Contexto-Enfermagem, 21, 708-716.

Gleriano, J. S., da Luz Zaiaz, P. C., Borges, A. P., Lucietto, G. C., Balderrama, P., de Almeida Corrêa, C. R., \& Chaves, L. D. P. (2019). Atenção integral na percepção dos profissionais da Estratégia Saúde da Família. Revista de Enfermagem UFPE on line, 13.

Guimarães, J. A. F., Aquino, P. S., Pinheiro, A. K. B., \& Moura, J. G. (2012). Pesquisa Brasileira sobre prevenção do câncer de colo uterino: uma revisão integrativa. Revista RENE, 13(1):220-230.

Júnior, A. R. F., de Sousa Albuquerque, R. A., Moreira, D. G., da Ponte, H. M. S., \& Rodrigues, M. E. N. G. (2019). Atuação do enfermeiro na visita domiciliar puerperal: perspectivas sobre o papel profissional. Revista Baiana de Saúde Pública, 43(3), 567-580.

Maciel, N. D. S., Luzia, F. J. M., Ferreira, D. D. S., Ferreira, L. C. C., Mendonça, V. D. M., Oliveira, A. W. N., \& Sousa, L. B. D. (2021). Busca ativa para aumento da adesão ao exame Papanicolaou. Rev. enferm. UFPE on line, 1-11.

Marqui, A. B. T. D. (2014). Endometriose: do diagnóstico ao tratamento. Rev. enferm. atenção saúde, 97-105.

Medeiros, P. F. D., \& Guareschi, N. M. D. F. (2009). Políticas públicas de saúde da mulher: a integralidade em questão. Revista Estudos Feministas, 17, 3148 .

Ohl, I. C. B., Ohl, R. I. B., Chavaglia, S. R. R., \& Goldman, R. E. (2016). Ações públicas para o controle do câncer de mama no Brasil: revisão integrativa. Revista Brasileira de Enfermagem, 69, 793-803.

Oliveira, I. G. D., Castro, L. L. D. S., Bezerra, R. A., Sousa, L. B. D., Santos, L. V. F. D., \& Carvalho, C. M. D. L. (2020). Identificação de fatores de risco à saúde entre mulheres usuárias de métodos contraceptivos hormonais. Rev. Pesqui.(Univ. Fed. Estado Rio J., Online), 786-792.

Silva, L. S. D., Leal, N. P. D. R., Pimenta, C. J. L., Silva, C. R. R. D., Frazão, M. C. L. O., \& Almeida, F. D. C. A. D. (2020). Contribuição do enfermeiro ao aleitamento materno na atenção básica. Rev. Pesqui.(Univ. Fed. Estado Rio J., Online), 774-778.

Silva, V. G. D., \& Ribeiro, P. M. (2020). Violência contra as mulheres na prática de enfermeiras da atenção primária à saúdea. Escola Anna Nery, 24.

Souza, M. T. D., Silva, M. D. D., \& Carvalho, R. D. (2010). Revisão integrativa: o que é e como fazer. Einstein (São Paulo), 8, 102-106.

Umpiérrez, A. F. (2013). La gestión del cuidado en la legislación profesional: algunas consideraciones para su regulación. Ciencia y Enfermería, 19(3), 103109.

Villela, W. V., \& Arilha, M. (2003). Sexualidade, gênero e direitos sexuais e reprodutivos. In Sexo \& vida: panorama da saúde reprodutiva no Brasil (pp. 95$150)$.

Wollstonecraft, M. (2017). Reivindicação dos direitos da mulher: edição comentada do clássico feminista. Boitempo Editorial, 1, (3), 256. 